\title{
Supporting Information for Publication: Realistic Cyclic Voltammograms from Ab Initio Simulations in Alkaline and Acidic Electrolytes
}

Jan Rossmeisl ${ }^{\bullet}$, Kim D. Jensen ${ }^{\bullet}$, Amanda S. Petersen, Logi Arnarson, Alexander Bagger, María Escudero-Escribano ${ }^{\circ}$

University of Copenhagen (UCPH), Department of Chemistry, Center for High Entropy Alloy

Catalysis, Universitetsparken 5, 2100 Copenhagen Ø, Denmark

Computational Methods and Model

The equations presented below utilizes a slightly different notation than in the Theory and Discussion section of the paper. Herein $N$ has been separated into $N_{\mathrm{H}}$ and $N_{I o n}$, thus distinguishing the elaboration from the computational hydrogen electrode (CHE) to the generalized CHE (GCHE) framework.

The GCHE potential has been calculated using the framework seen below:

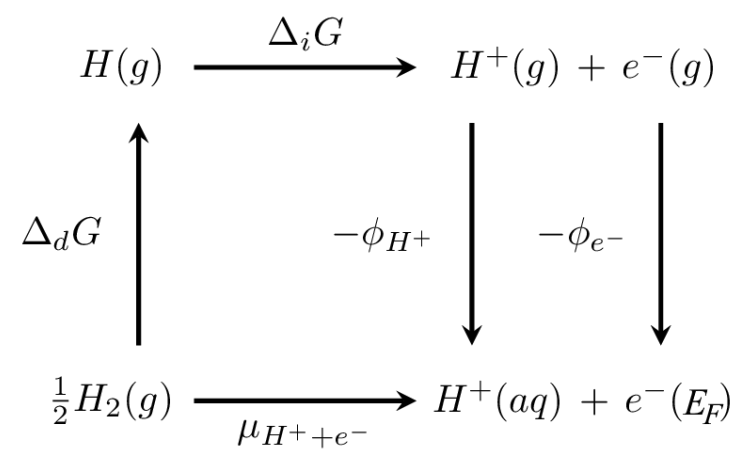

The chemical potential of protons has been defined using the Born-Haber cycle

$$
\mu\left(\mathrm{H}^{+}+\mathrm{e}^{-}\right)=\Delta_{d} G+\Delta_{i} G-\phi_{\mathrm{H}^{+}}-\phi_{e^{-}}
$$

where $\Delta_{d} G$ is the dissociation energy of hydrogen, $\Delta_{i} G$ is the ionization energy of hydrogen and $\phi_{\mathrm{H}^{+}}$and $\phi_{e^{-}}$are the workfunction for protons and electrons, respectively. Note the electron 
workfunction is defined through the Fermi level as $\phi_{e^{-}}=E_{e^{-}}-E_{E_{F}}$. At $\mathrm{pH} 0$ and zero applied potential $\left(U_{R H E}=0 \mathrm{~V}\right)$ this should be

$$
0=\Delta_{d} G+\Delta_{i} G-\phi_{\mathrm{H}^{+}}^{0}-\phi_{\mathrm{SHE}}
$$

The workfunction of the proton's $\mathrm{pH}$ dependence is expressed as

$\phi_{\mathrm{H}^{+}}=\phi_{\mathrm{H}^{+}}^{0}+2.3 k_{B} T \mathrm{pH}$

Now one is able to combine eq. (S1-S3) to yield

$$
\phi_{\mathrm{H}^{+}}=\Delta_{d} G+\Delta_{i} G+\phi_{\mathrm{H}^{+}}-\phi_{\mathrm{H}^{+}}^{0}-\phi_{\mathrm{SHE}}
$$

and

$$
\mu\left(\mathrm{H}^{+}+\mathrm{e}^{-}\right)=\phi_{\mathrm{SHE}}-\phi_{e^{-}}-2.3 k_{B} T \mathrm{pH}=-e U_{\mathrm{RHE}} .
$$

Note SHE, RHE and workfunction potentials are related by

$$
-e U_{\mathrm{RHE}}=\phi_{\mathrm{SHE}}-\phi_{e^{-}}-2.3 k_{B} T \mathrm{pH},
$$

and

$$
-e U_{\mathrm{SHE}}=\phi_{\mathrm{SHE}}-\phi_{e^{-}} .
$$

The above allows one to define the CHE potential ${ }^{1}$

$$
G\left(N, U_{\mathrm{RHE}}\right)=E(N)-E(N=0)-N \frac{1}{2} \mu_{\mathrm{H}_{2}}^{0}-N e U_{\mathrm{RHE}} .
$$

Using the above the GCHE potential can be calculated by adding/subtracting hydrogen atoms from the interface of interest, as shown below

$$
\begin{aligned}
G_{i}\left(N_{\mathrm{H}}, \phi_{\mathrm{e}^{-}}, \mathrm{pH}\right) & =E\left(N_{\mathrm{H}}, \phi_{e^{-}}\right)-\left\langle E\left(N_{\mathrm{H}}=0\right)\right\rangle \\
& -N_{\mathrm{H}_{2}} \frac{1}{2} \mu_{\mathrm{H}_{2}}^{0}-e N_{\mathrm{H}}\left[\phi_{\mathrm{SHE}}-\phi_{e^{-}}-2.3 k_{B} T \mathrm{pH}\right]
\end{aligned}
$$

For a generalized computational ion electrode ions are likewise able of being added and subtracted from the interface and similarly yields

$$
G_{i}\left(N_{\mathrm{H}}, N_{\text {Ion }}, \phi_{e^{-}}, \mathrm{pH}\right)=E\left(N_{\mathrm{H}}, N_{\text {Ion }}, \phi_{e^{-}}\right)-\left\langle E\left(N_{\mathrm{H}}=0, N_{\text {Ion }}=0\right)\right\rangle
$$




$$
\begin{aligned}
& -N_{\mathrm{H}^{\frac{1}{2}}} \frac{1}{\mathrm{H}_{2}}-e N_{\mathrm{H}}\left(\phi_{\mathrm{SHE}}-\phi_{e^{-}}-2.3 k_{B} T \mathrm{pH}\right) \\
& -N_{\text {Ion }} \mu_{\text {Ion }(s)}^{0}-e N_{\text {Ion }}\left[\phi_{\text {Ion }}-\phi_{e^{-}}-2.3 k_{B} T \ln \left(\frac{M_{\text {Ion }}}{M_{\text {Ion }}^{0}}\right)\right],
\end{aligned}
$$

where $N_{I o n}$ is the number and $M_{I o n}$ is the concentration of ions and $M_{I o n}^{0}$ is the standard condition of $1 \mathrm{M}$. The Boltzmann averaging of the energies have been evaluated in the following manner

$$
G=\frac{1}{Z} \sum_{i=1}^{Z} G_{i} \exp \left(\frac{G_{i}\left(N_{\mathrm{H}}, N_{I o n}, \phi_{\left.e^{-}, \mathrm{pH}\right)}\right.}{k_{B} T}\right)
$$

Using the framework presented above one is able to define GCHE of any interface factoring in ions, $\mathrm{pH}$ and applied interface potential.

In Figure 5 and 7 and all conversions between unit cell and experimental areas it should be noted that the charge per area/unit cell of $\operatorname{Pt}(111)$ has been evaluated considering a pristine (111)-face centered cubic (FCC) surface with a lattice constant of $4.017 \AA$. This lattice constant has been estimated from $a b$ initio relaxation simulations and corresponds reasonable with surface vacuum values of 3.9244 A. ${ }^{2}$

\section{Implementation}

The values for $\phi_{\mathrm{H}^{+}}$and $\phi_{\mathrm{e}^{-}}$can be calculated using the appropriate potential values and noting the workfunction at zero for the standard hydrogen electrode, i.e. on the standard hydrogen scale is -4.44 $\mathrm{eV}$ at $\mathrm{pH} 0 .{ }^{3}$ The workfunction at zero potential at higher $\mathrm{pH}$ values are shifted by $0.059 \mathrm{eV} / \mathrm{pH}$ following the Nernst equation. ${ }^{4}$ By using this measured value simulations describing the bringing of the protons through the vacuum/water interface and estimating the energy of a proton suspended in water is avoided.

$E_{N}\left(\phi_{e^{-}}\right)$can be obtained by sampling different structures of the electrolyte, e.g. by using molecular dynamics. If all structures of the electrolyte molecules can be visited in a representative manner such a simulation should result in a normal distribution with the workfunction as the order parameter. There are many microstates for which the dipoles of the electrolyte molecules approximately cancel perpendicular to the surface and only a few states where all the dipoles are pointing in a specific direction. This has schematically been shown in Figure S1. 


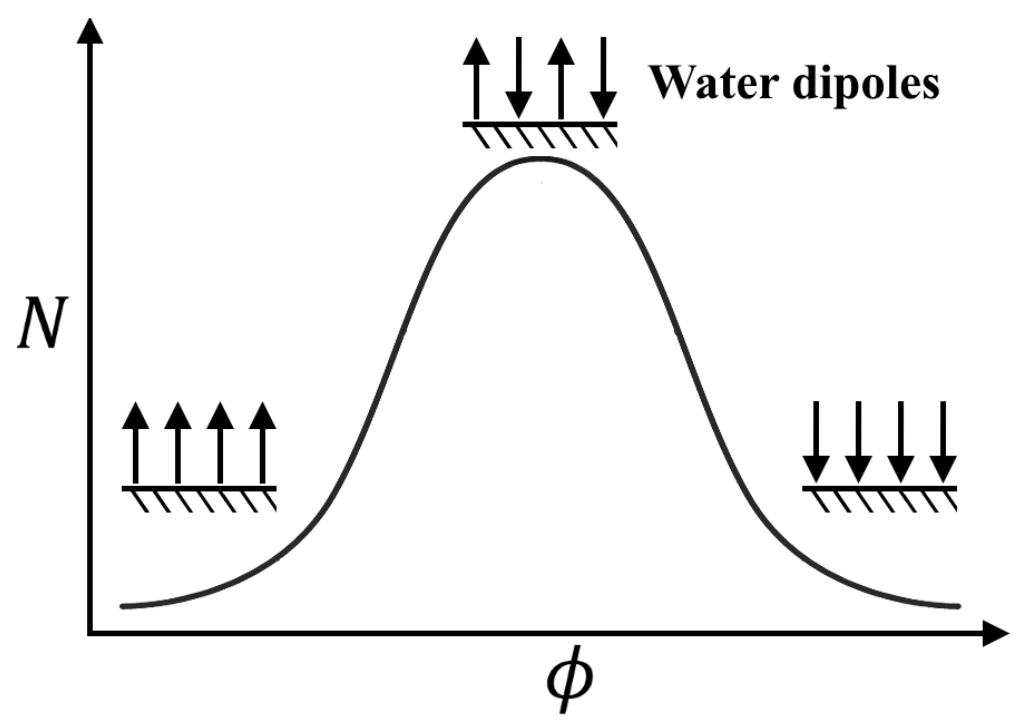

Figure S1. Schematic representation of a distribution of number of "snapshot" structures as function of the workfunction. Note higher entropy states are more probable.

This sampling of microstates is done for a different number of hydrogen atoms in the interface. When the normal distribution is obtained for each number of hydrogen atoms, simulated in the interface, these can then be normalized. Thereby, the total number of microstates corresponding to each number of hydrogen atoms remain the same. This corresponds to the assumption, that there is no contribution to the configurational entropy by adding or subtracting hydrogen from the interface. If the proton ends up adsorbed on the surface or in the Helmholtz layer this is a fair assumption. However, it is not valid if the proton ends up in the bulk of the electrolyte, where the configurational entropy is related to the $\mathrm{pH}$. The simulations can only contain a limited amount of water perpendicular to the surface so the proton cannot approach bulk electrolyte. This means that the assumption cannot be valid if the unit cell of the simulation eventually involves bulk electrolyte. Furthermore, the change of entropy of the water molecules upon adding or removing hydrogens or ions is not included. It could be important to make estimates for the change in entropy for a direct comparison to experiments, hopefully some general/systematic values can be obtained.

$N$, the number of hydrogen atoms are sampled in intervals of coverages which is determined by the area of the simulation cell, the larger the area the better the sampling. We note that the shape of the normal distribution also changes with area, the larger the area of the simulation cell gives a sharper distribution of microstates. This could lead to the misconception that a constraint is missing in the method, but that is not the case. All conclusions turn out to be independent on the size of the 
simulation cell because the sampling becomes correspondingly better, meaning that the change in dipole moment of the interface also becomes smaller upon adding a hydrogen atom.

The Boltzmann distribution of structures can be obtained in small bins of the potential in the phase diagrams. All structural properties of the interface are when calculated as Boltzmann weighted averages, over the structures within the given potential bin. The molecular dynamics is done at constant extensive variables and the forces are not subject to the constraints that should be imposed by constant intensive variables. However, the GCHE evaluates the energy of the structure at constant intensive variables and molecular dynamics is only used as a manner of sampling structures. The trajectories are not important per se.

If a charged molecule is adsorbed on the electrode surface and the electrons are transferred it is coupled proton/electron transfer, then the molecule and the counter electron is at the same potential and the energy become independent on the workfunction: $E_{N}\left(\phi_{e^{-}}\right)-\mu N=E_{N}-\mu N$ and the charge is $N$, this is the situation depicted in Figure 1. However, if no electrons are transferred to the counter electrons and the charged molecule are not at the same potential, then $E_{N}\left(\phi_{e^{-}}\right)$is a linear function of $N \phi_{e^{-}}$or $N \mu$ and thereby $\gamma_{N}$ becomes independent on $\mu$ and the charge transferred is zero.

\section{Electrochemical Methods}

All $\operatorname{Pt}(111)($ MaTecK, $99.995 \%, h \times \emptyset=4 \times 5 \mathrm{~mm})$ were prepared by utilizing the Clavilier method $^{5}$ in which the Pt(111) crystal was heated using a flame torch (Proxxon, liquid petroleum torch) and annealed minimum three times and cooled in an 1:1 $\mathrm{Ar} / \mathrm{H}_{2}(\mathrm{~N} 5 / \mathrm{N} 4.5)$ atmosphere Figure $\mathrm{S} 2$. 


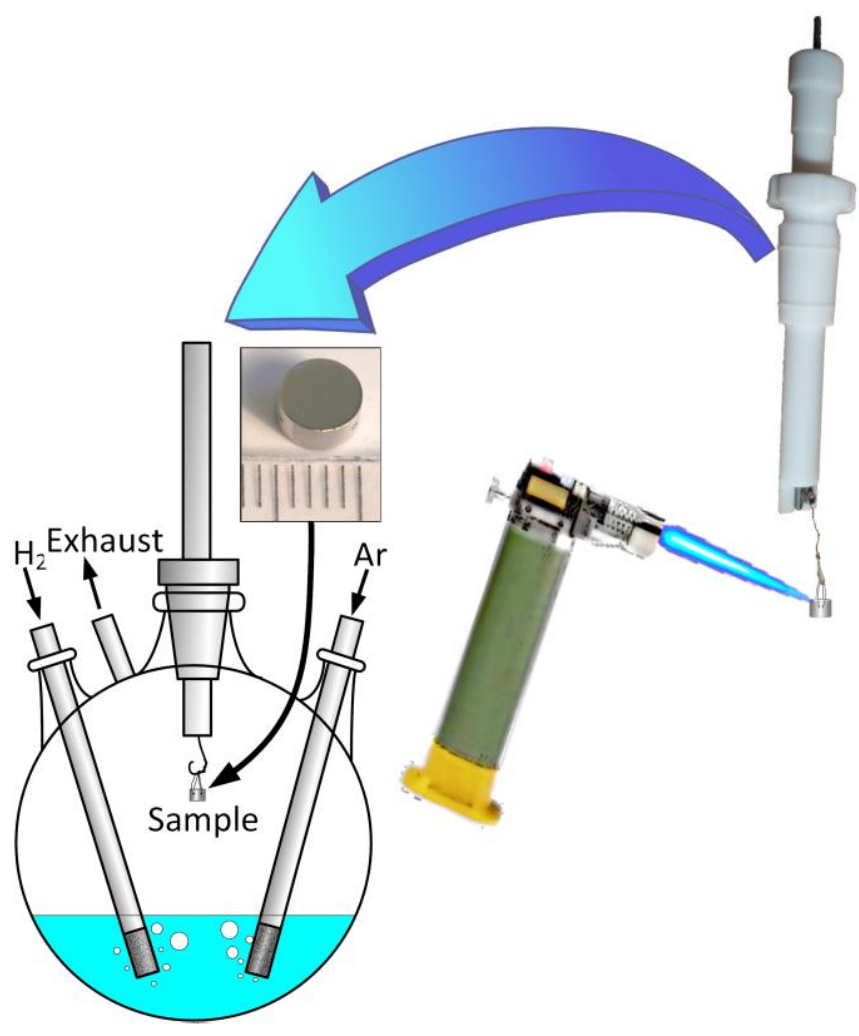

Figure S2. Scheme for preparing Pt(111) crystals; Pt(111) is annealed and cooled in a glass cell with $\mathrm{Ar} / \mathrm{H}_{2}$ atmosphere. Adapted from ${ }^{6}$.

Crystals were then mounted in a rotating ring (disk) electrode (R(R)DE) setup (Pine instrument) and submerged in a three-electrode cell under potential control at ca. $0.1 \mathrm{~V}$ vs. RHE. ${ }^{7}$ All glassware (cell etc.) had been cleaned in piranha $\left(1: 3 \mathrm{H}_{2} \mathrm{O}_{2}\right.$ and $\left.\mathrm{H}_{2} \mathrm{SO}_{4}\right)$ for $24 \mathrm{~h}$ and rinsed five times afterwards before additionally being rinsed 5-10 times while heated to approximately $89{ }^{\circ} \mathrm{C}$ for 15 min between each rinse. ${ }^{6}$

Electrochemical measurements were all done in either $\mathrm{NaOH}$ (Merck, Suprapur®) or $\mathrm{HClO}_{4}(\mathrm{Merck}$, $70 \%$, Suprapur ${ }^{\circledR}$ ) with or without $\mathrm{NaClO}_{4}$ (Sigma-Aldrich, Trace metal basis 99.99\%). All CVs were done at $50 \mathrm{mV} / \mathrm{s}$ at room-temperature in Ar-saturated electrolytes at rotations of $400 \mathrm{rpm}$. Ohmic drop compensation and HOR/HER evaluation of RHE potential was conducted for all measurements. ${ }^{6,8}$

All electrochemical measurements were conducted in a three electrode cell presented in earlier work, ${ }^{6,9}$ see Figure S3. 


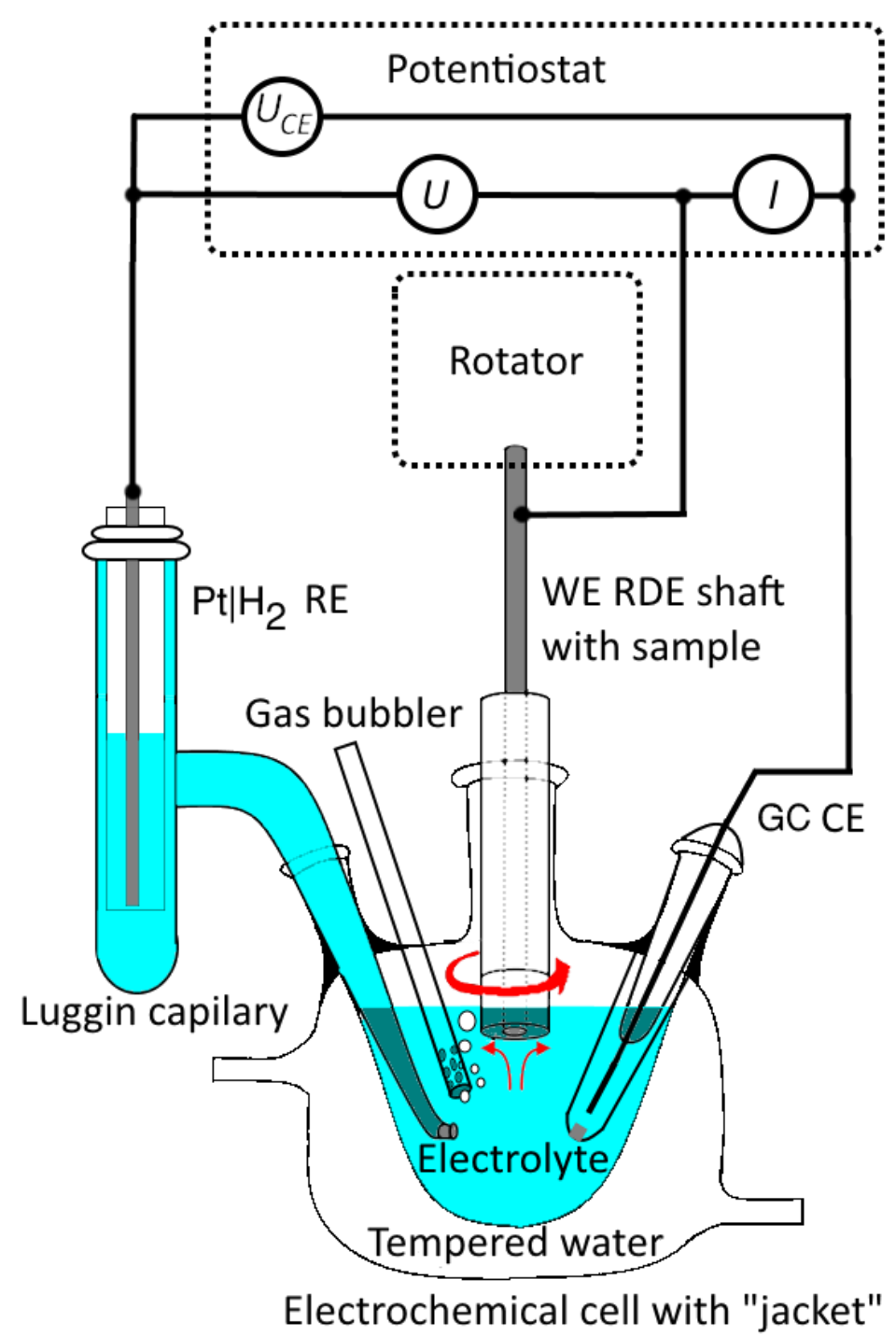

Figure S3. Schematic of three-electrode cell configuration used for the experiments, adapted from ${ }^{9}$.

Hanging meniscus CVs in different $\mathrm{NaOH}$ and concentrated electrolytes were collected to account for the effect of changes to either $\left[\mathrm{Na}^{+}\right]$or $\left[\mathrm{ClO}_{4}^{-}\right]$concentrations on the $\operatorname{Pt}(111)$ surface, see Figure S4. 

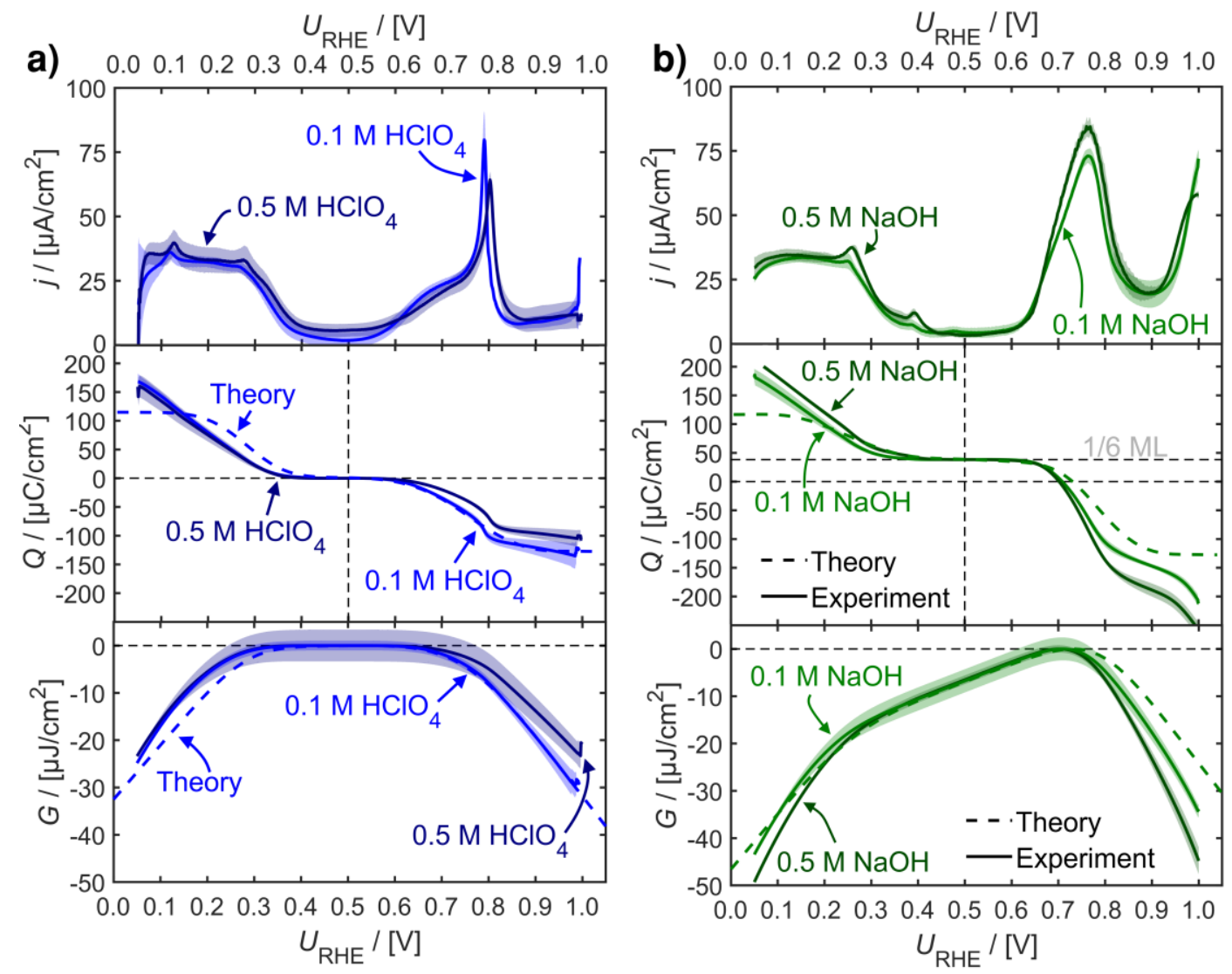

Figure S4. Anodic scan of Pt(111) base CVs (top), corresponding charge isotherms (middle) and phase diagrams; a) In $0.1 \mathrm{M} \mathrm{HClO}_{4}$ (blue) and $0.5 \mathrm{M} \mathrm{HClO}_{4}$ (dark blue); b) In $0.1 \mathrm{M} \mathrm{NaOH}$ (green) and $0.5 \mathrm{M} \mathrm{NaOH}$ (dark green); Taken at $50 \mathrm{mV} / \mathrm{s}$ at room-temperature. Measurements in $0.5 \mathrm{M}$ electrolytes were taken in a hanging meniscus configuration whereas $0.1 \mathrm{M}$ data was obtained at 400 rpm in an RDE setup.

From Figure $\mathrm{S} 4$ it is noted that $i$ ) equivalent base $\mathrm{CVs}$ can be obtained in RDE and hanging meniscus configurations; only at low potentials small changes due to HOR/HER are likely to occur. ii) $\mathrm{OH}$ coverage seems to increase (i.e. $\mathrm{OH}$ weakening) when increasing $\left[\mathrm{Na}^{+}\right]$. iii) Conversely $\mathrm{OH}$ coverage seems to be suppressed (e.g. $\mathrm{OH}$ blocking) when increasing $\left[\mathrm{ClO}_{4}^{-}\right]$. This suggests an intricate relationship between both $\left[\mathrm{Na}^{+}\right]$and $\left[\mathrm{ClO}_{4}^{-}\right]$relative to the interface energy structures. Following eq. S10 this is hardly surprising as one would expect the shift in energy by adding cations and anions to follow

$$
E \propto 2.3 k_{B} T \mathrm{pH}-k_{B} T \log \left(\left[\mathrm{Na}^{+}\right]\right)-k_{B} T \log \left(\left[\mathrm{ClO}_{4}^{-}\right]\right)
$$


To uncover whether changes in surface structures can be described solely by $\mathrm{pH}$ and ion concentrations a range of measurements were conducted in $0.1 \mathrm{M} \mathrm{HClO}_{4}$ (fixed pH) and $x \mathrm{M} \mathrm{NaClO}_{4}$ Something which also has been attempted by others ${ }^{10}$, see Figure S5.

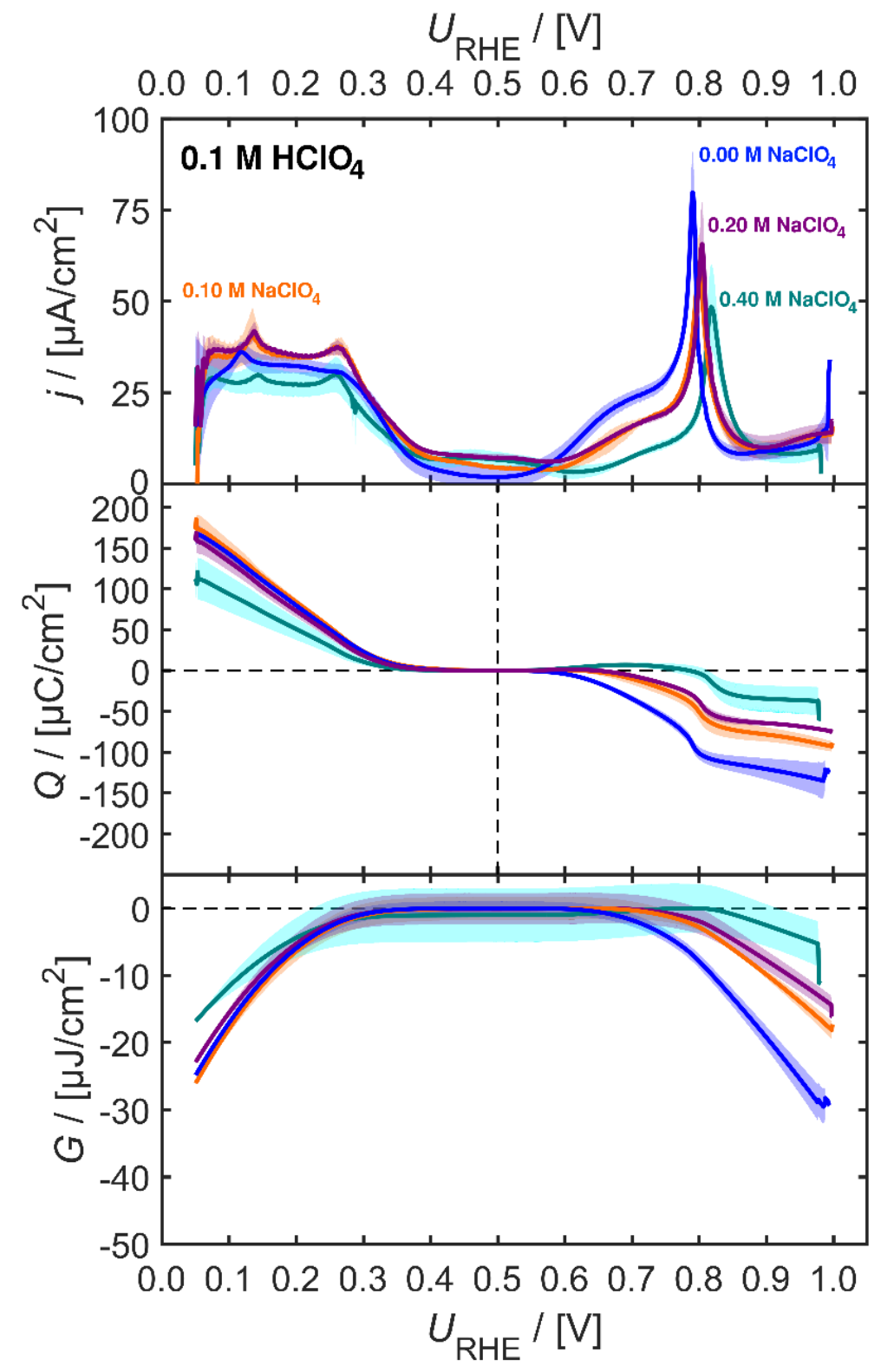

Figure S5. Anodic scans of Pt(111) base CVs (top), corresponding charge isotherms (middle) and phase diagrams (bottom); Data obtained in either pure $0.1 \mathrm{M} \mathrm{HClO}_{4}$ (blue) or mixed with $0.1 \mathrm{M}$ (orange), $0.2 \mathrm{M}$ (purple) or $0.4 \mathrm{M}$ (teal) $\mathrm{NaClO}_{4}$. Taken at $50 \mathrm{mV} / \mathrm{s}$ at room-temperature. Measurements in pure $\mathrm{HClO}_{4}$ and with $0.1 \mathrm{M} \mathrm{NaClO}_{4}$ were obtained in an $\mathrm{RDE}$ setup (400 rpm) the remaining were taken in a hanging meniscus configuration. 
Comparing Figure S5 with S4 it is clear, that there is a substantial difference between measuring $\mathrm{Pt}(111)$ in $0.5 \mathrm{M} \mathrm{HClO}_{4}$ and $0.1 \mathrm{M} \mathrm{HClO}_{4}+0.4 \mathrm{M} \mathrm{NaClO}_{4}$, even though $\mathrm{pH}$ and $\left[\mathrm{ClO}_{4}^{-}\right]$is maintained more or less the same ( $\mathrm{pH} 0.3$ vs. 1.0, repsectively). Either $\mathrm{Na}^{+}$is able to influence measurements in acid (see Figure 5) or $i$ ) $\mathrm{NaClO}_{4}$ dissociation is more likely than that of $\mathrm{HClO}_{4}$ or $i$ ) the $\mathrm{NaClO}_{4}$ salt used in the experiments is not pure enough for this kind of analysis. Given that the $\mathrm{NaClO}_{4}$ is "only" 99.99\% pure and any $\mathrm{MCl}$ or $M \mathrm{NO}_{x}$ compounds incorporated into the salt even in ppm-levels will have a detrimental effect on the resulting CVs one should be careful concluding too much from Figure S5. ${ }^{11}$ Hence, from the experiments with the $\mathrm{NaClO}_{4}$ salt we are unable to say anything about the specific effect(s) on the coverage except that it surely do has some effect. Moreover, $\mathrm{Na}^{+}$adsorption may in fact also dominate the $\mathrm{H}$ region under acidic conditions as we later illustrate in Figure S11.

Many, ${ }^{10,12-18}$ besides us in this work, have attempted to probe the effect of ions in relation to $\mathrm{pH}$ following simple relationships such as that proposed in eq. (S12). However, the approach has often been to probe the $\mathrm{pH}$ by maintaining the ionic strength. E.g. dilution of $\mathrm{HClO}_{4}$ and adding ions such as $\mathrm{NaClO}_{4}, \mathrm{KClO}_{4}, \mathrm{NaF}$ or alternatively using buffers. However, it is by now clear that 1) the $\mathrm{ClO}_{4}{ }^{-}$ concentration will influence the CV response. 2) Adding additional cations and anions will allow for new "lowest energy" interface structures i.e. disallowing direct comparison between CVs when changing electrolytes. A major obstacle for these ${ }^{10,12-18}$ kind of studies is that alkaline media tend to include cations $(\mathrm{NaOH}, \mathrm{KOH}$, etc.) and thus $\mathrm{pH}$ and cation concentration cannot be probed independently. Moreover, pure dilution of both acids and alkaline media is restricted in the range in which stable CVs can be obtained (e.g. [acid] $\vee$ [alkaline] $>1 \mathrm{mM}$ ), as too neutral $\mathrm{pH}$ will tend to change adsorption mechanisms due to the increasingly slow kinetics. Thirdly, cation and anion adsorption is in of itself $\mathrm{pH}$ dependent i.e. an $\mathrm{OH}$ adsorption features in one electrolyte that appears similar in another electrolyte may be due to both $\mathrm{OH}$ and anion adsorption in this instance. To illustrate this issue eq. (S12) has been used under the assumption that one is able to make electrolyte of $1 \mathrm{mM} \leq[\mathrm{NaOH}] \leq 1 \mathrm{M}$ alkaline solutions or $1 \mathrm{mM} \leq\left[\mathrm{HClO}_{4}\right] \leq 1 \mathrm{M}$ acidic solutions with varying amounts of sodium perchlorate $\left(0 \mathrm{M} \leq\left[\mathrm{NaClO}_{4}\right] \leq 1 \mathrm{M}\right)$. Figure $\mathrm{S} 6$ reveals the expected changes in energetics as of eq. (S12), that one would expect from such electrolyte-space. 

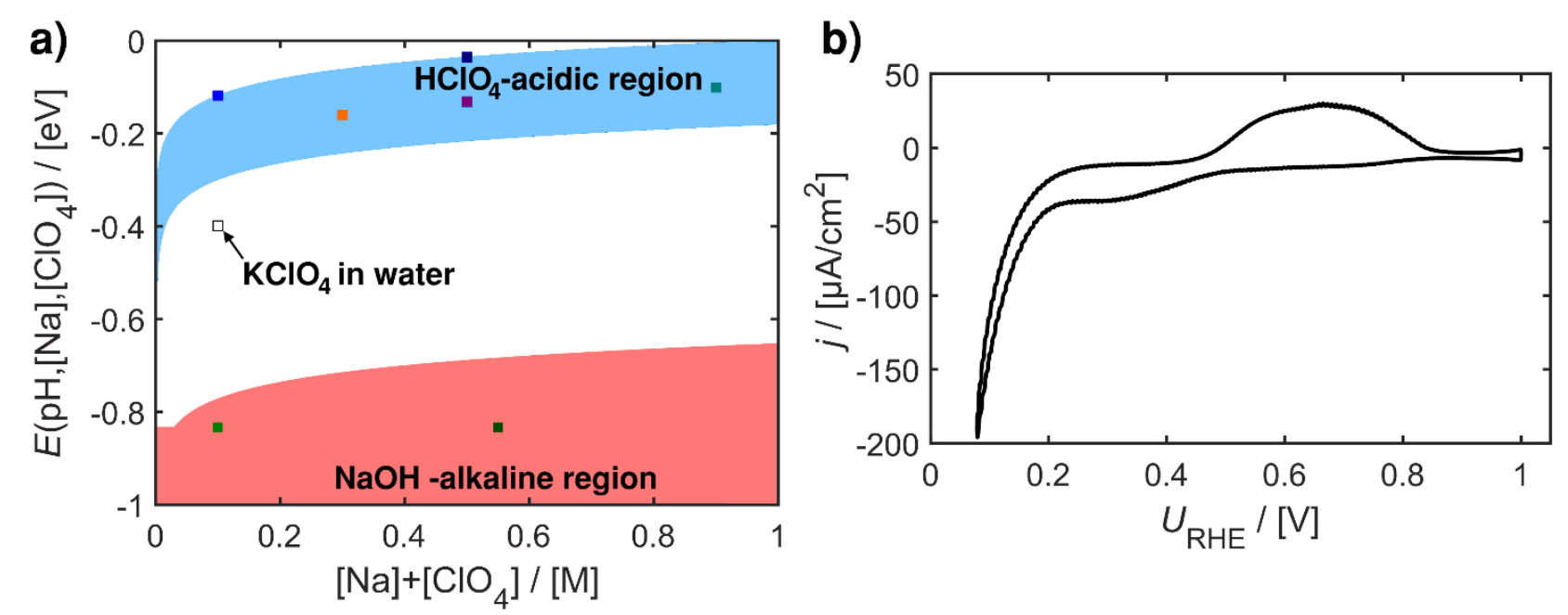

Figure S6. a) The shaded area designates the electrolyte-energetics space one is able to probe experimentally using eq. (S12) and the concentrations restrictions $1 \mathrm{mM} \leq[\mathrm{NaOH}] \leq 1 \mathrm{M}$ alkaline solution or $1 \mathrm{mM} \leq\left[\mathrm{HClO}_{4}\right] \leq 1 \mathrm{M}$ acidic solution with varying amounts $0 \mathrm{M} \leq\left[\mathrm{NaClO}_{4}\right] \leq 1 \mathrm{M}$. This energy-electrolyte space have been plotted relative to the accumulated $\mathrm{Na}^{+}$and $\mathrm{ClO}_{4}{ }^{-}$ concentrations. Colored points correspond to the electrolytes studied in Figure 7, S4, S5 and S8. Moreover, a single point for $0.1 \mathrm{M} \mathrm{KClO}_{4}$ has been added; b) Ar-saturated $0.1 \mathrm{M} \mathrm{KClO}_{4} \mathrm{CV}$ taken at $50 \mathrm{mV} / \mathrm{s}$ at room-temperature in a hanging meniscus configuration.

From Figure S6a it is obvious that there is a vast region in the electrolyte space which cannot be easily probed experimentally by adding salts into the electrode-electrolyte interface. Furthermore, attempting to reach the region may result in $\mathrm{CVs}$, as that in Figure $\mathrm{S} 6 \mathrm{~b}$ of $\mathrm{Pt}(111)$ in $0.1 \mathrm{M} \mathrm{KClO}_{4}$, where slow adsorption/desorption kinetics hinders CV comparison.

Note the rather neutral electrolyte ( $\mathrm{pH} 4.7 \pm 0.3$ ) of Figure $\mathrm{S} 6 \mathrm{~b}$ seems to shift potentials $v s$. RHE in a puzzling fashion. The reason for the anomalous HER relative to the potential is not understood yet, but the results were reproducible over six different experiments with 0.10-0.05 $\mathrm{M} \mathrm{KClO}_{4}$. With that we are not stating we are able to produce $\mathrm{H}_{2}$ above the RHE potential but rather, the RHE electrode utilized is unstable under these neutral conditions.

Typical Fitting Procedure

To evaluate the difference between the AIMD modelled interface energies and the experimentally observed data the following fitting approach, see Figure S7, was utilized. 

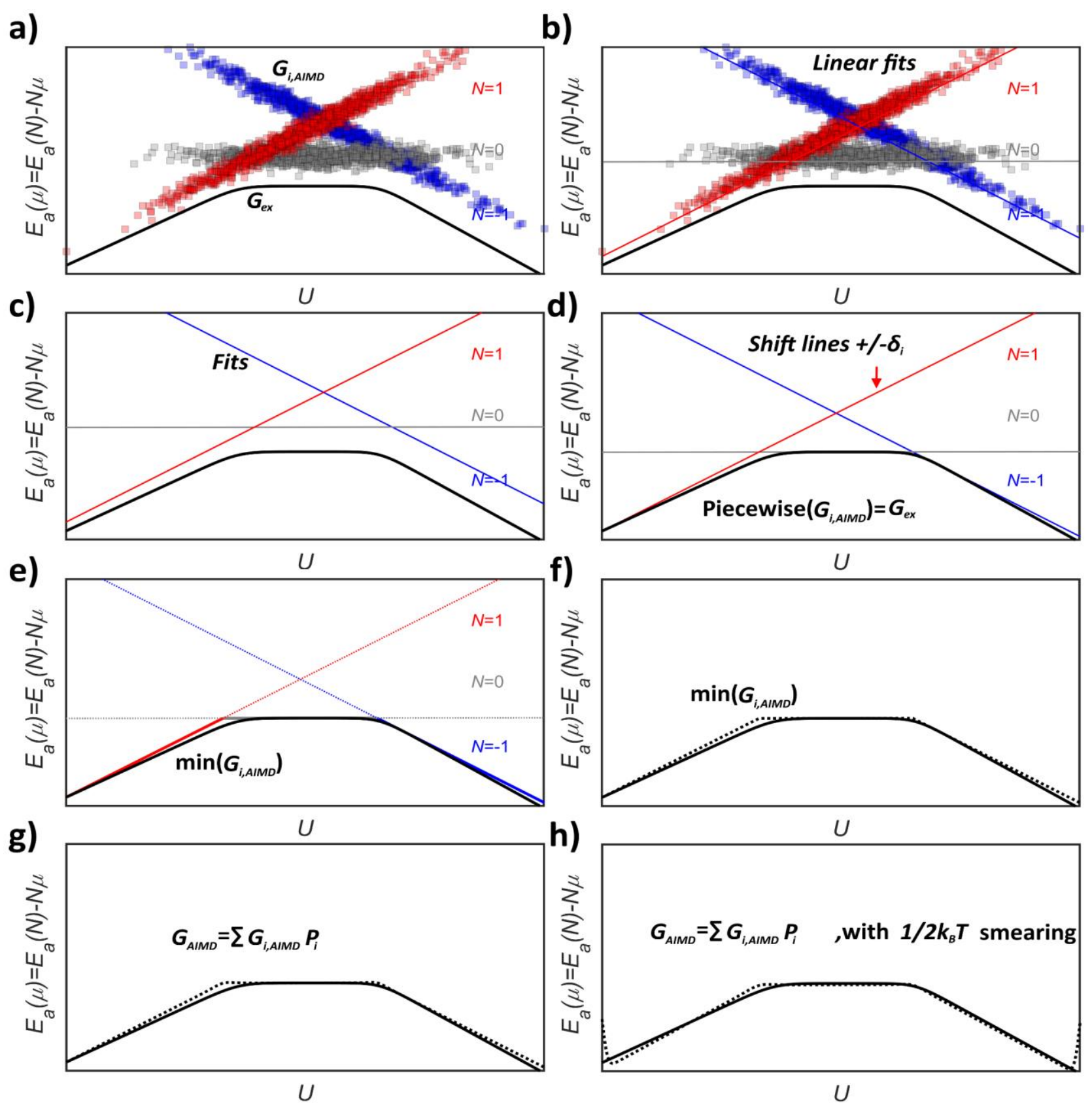

Figure S7. Scheme for fitting AIMD data to experimental data; a-b) Steps in the utilized fitting procedure.

As seen in Figure S7a, experimental interface energy data can be derived. This has in our case been evaluated assuming a specific stable structure being known at a specific potential, e.g. water dominating the $\operatorname{Pt}(111)$ electrode in acid in the double-layer region etc. This can be co-plotted together with the AIMD data. Next (see Figure S7b) it is straight forward to represent all the specific AIMD states as simple linear fits describing the expected binding as function of potential. Note, whether one choses to utilize all the data or just some lower percentile of the available states does not 
matter greatly in the fitting procedure (as long as it is done so with no change in weighting across the entire potential range). Such selective use of data only influences the magnitude of the shift required for the fit. We found it beneficial to reduce the fitting problem to the fitting of these theoretical energy structure lines and not the individual states, see Figure S7c, although this is equally possible. After this simplification of the fitting problem one may evaluate the energy needed to shift the energy structure lines to fit with the experimental lines. This piecewise process relies on finding the shift required for a specific structure energy line (with a specific slope) to tangent the experimental values. Essentially one finds the point where the experimental charge isotherm equals the energy structure line's slope and evaluate the shift required, see Figure S7d. Once completed the main part of the fitting procedure is done.

In Figure S7 an example of three different states are seen competing on a surface. To evaluate the actual expected continuum of Gibbs free energies/interface energies one introduces a weighting, i.e. the lowest energy structure at a given potential are expected to dominate. Hence, one finds the minimum expected interface energy structure, seen in Figure S7e,f. Firstly, the part of the AIMD data evaluated to be the minimum energy the associated structure is found, highlighted in Figure S7. Secondly, this lowest energy structure is set aside, plotted in Figure S7f. Now we are able to find the fitted AIMD data structure by simply adding up all the states of the energies at a given potential and multiply each state with the probability that said state dominates at that specific potential, this has been done in Figure S7g where

$$
G_{A I M D f i t}(U)=\sum G_{i, A I M D}(U) P_{i}
$$

and in the Figure S7g example $P_{i}=p_{i}$

$$
p_{i}=\frac{\exp \left(-\frac{G_{i, A I M D}-\min \left(G_{i, A I M D}\right)}{k_{B} T}\right)}{\sum \exp \left(-\frac{G_{i, A I M D}-\min \left(G_{i, A I M D}\right)}{k_{B} T}\right)}
$$

Generally, the probability can be given as the product of any $j \in i$ normalized distributions

$$
P_{j}=\prod p_{j}
$$

Hence, one can besides the Boltzmann weighting in eq. (S14), which is the minimum requirement, also include normal distributed smearing, seen exemplified in Figure S7h. The fitting procedure has 
been utilized both on the acid and alkaline data, as seen in Figure 7 and also here in S8 in which the raw AIMD 2D phase diagram, charge isotherm and simulated CVs are shown with experimental data.

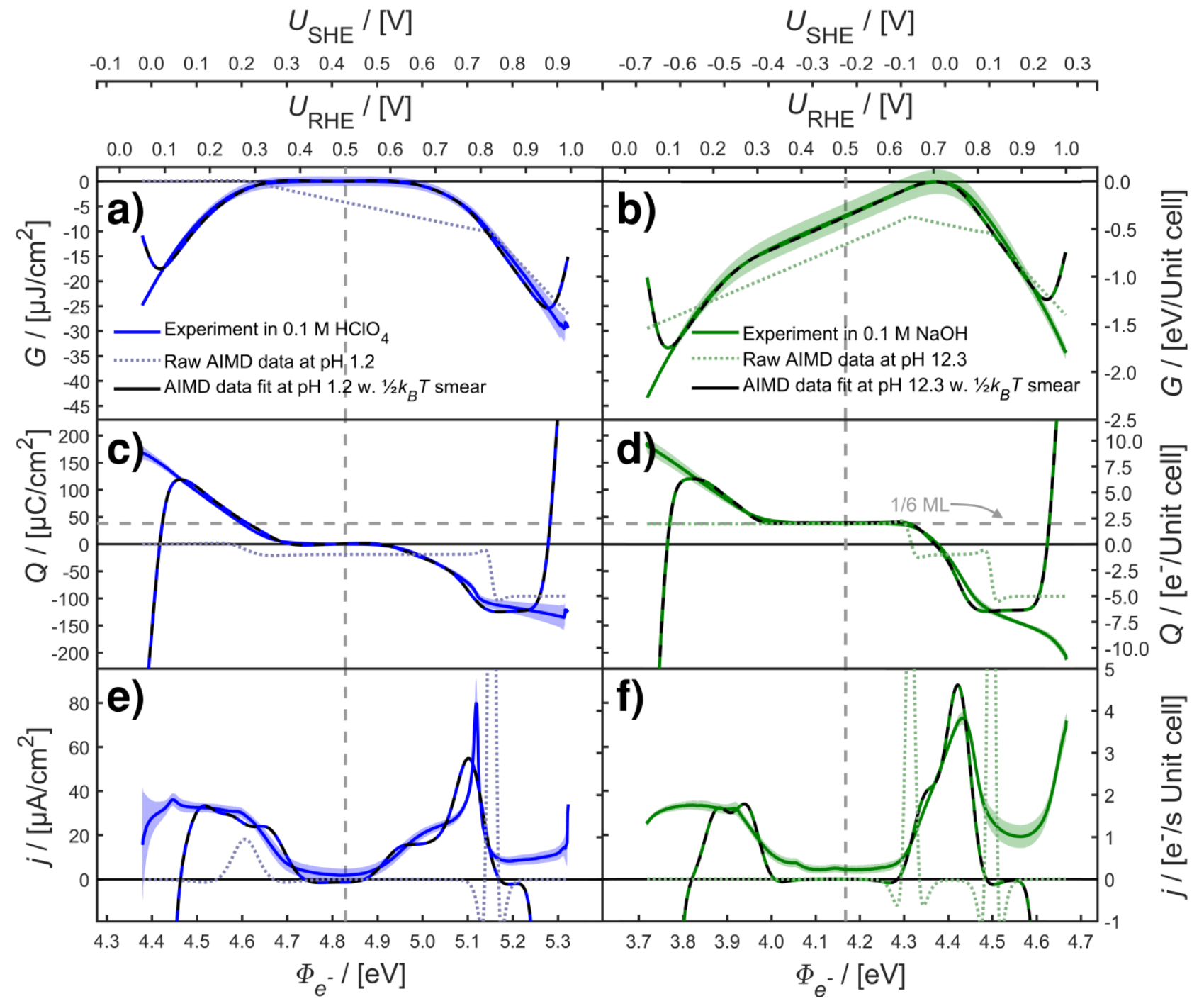

Figure S1. AIMD simulations with and without corrections co-plotted with corresponding experimental data of $\operatorname{Pt}(111)$; a,c,e) Under acidic conditions at pH 1.2 (blue); b,d,f) Under alkaline conditions at pH 12.3 (green); Experimental data is based on eight distinct measurements each in 0.1 $\mathrm{M} \mathrm{HClO}_{4}$ and in $0.1 \mathrm{M} \mathrm{NaOH}$ (error correspond to one standard mean of deviation). All experiments were obtained at room-temperature, $50 \mathrm{mV} / \mathrm{s}, 400 \mathrm{rpm}$ and in Ar-saturated electrolytes; a,b) Free energy diagrams based on the Boltzmann weighted minimum states of Figure 6 with and without fit corrections co-plotted with experimental data from doubly integrated anodic sweep CVs; c,d) Corresponding simulated and experimental charge isotherms; e,f) Corresponding simulated and experimental anodic sweeps of base CVs. 
From the preceding it becomes obvious that a fit can be done ad hoc without the AIMD data, as long as a coverage at a certain potential is known. One can just include lines with the slopes expected for the system. To showcase this; the case of the experimental interface energy structures in acid and alkaline have been fitted. In acid structure lines with 1-8H atoms are considered (lines with slopes 1 to 8) together with structures of 1-7OH's (having slopes of -1 to -7) and of course water (slope zero). In alkaline the same structures are considered. However additionally, structures with $2 \mathrm{Na} 0-5 \mathrm{H}$ atoms are considered (slopes of 2-7). Note, that as $2 \mathrm{Na} N \mathrm{H}$ dominates, rather than structures consisting of pure hydrogen, the fit corrections in acid have also been uncritically employed in the case of $\operatorname{Pt}(111)$ in alkaline media. In Figure S9 the fits (following the guidelines of Figure S7) have been shown.
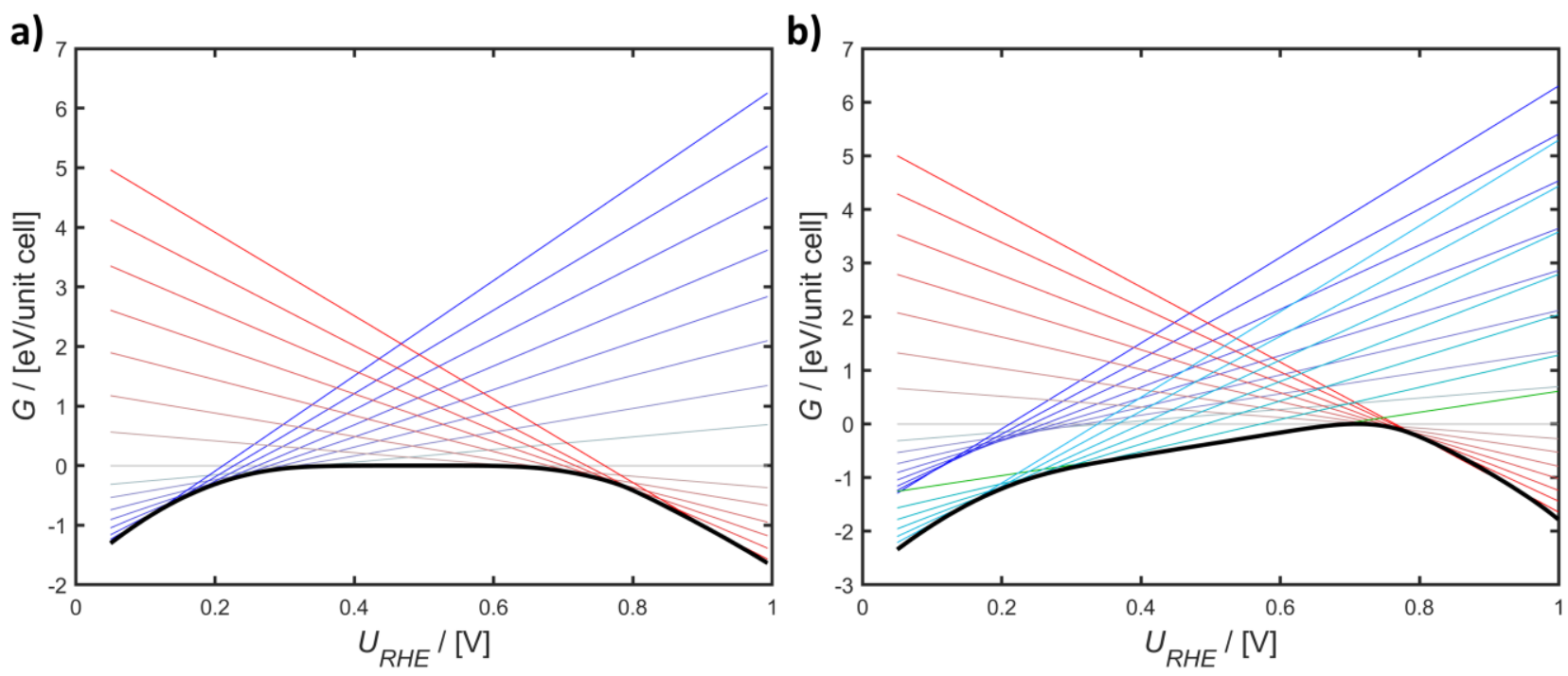

Figure S9. Fitting of virtual energy structure lines to experimental Pt(111) data (400 rpm, roomtemperature, $50 \mathrm{mV} / \mathrm{s})$; a) In $0.1 \mathrm{M} \mathrm{HClO}_{4}$; b) In $0.1 \mathrm{M} \mathrm{NaOH}$.

Using the fits in acid and alkaline one can highlight, by subtracting same energy structure line in acid and alkaline from one another, that $\mathrm{OH}$ and $\mathrm{H}$ (that latter only implicitly) adsorb at the same RHE potential, see Figure S10. 


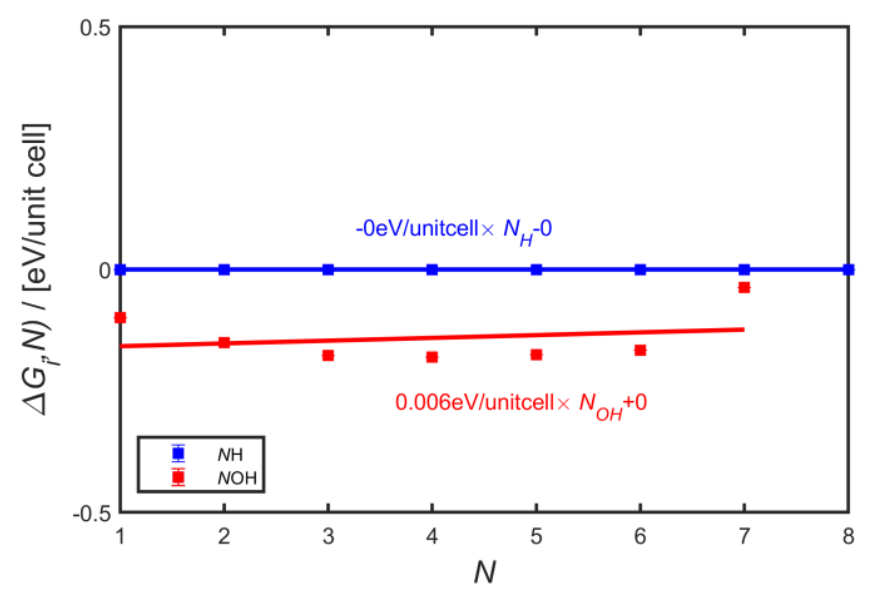

Figure S10. Subtracting of fitted interface energy lines in acid and alkaline, with 1-8H (blue) and 1$7 \mathrm{OH}(\mathrm{red})$; Note as same $\mathrm{H}$ fit has been used in acid and alkaline the difference in fitted lines is zero.

Using the fitting procedure and including Na structures under acidic conditions, it becomes clear that the main difference in adsorption structures in acid and alkaline may be attributed to the double layer coverage of water in the acid while in alkaline $\mathrm{Na}+$ structures dominates, see Figure $\mathrm{S} 11$. However, in the case of $0.1 \mathrm{M} \mathrm{HClO}_{4}$ with some $\mathrm{Na}$ present and in the case of $0.1 \mathrm{M} \mathrm{NaOH}$, we expect the $\mathrm{H}_{\text {UPD }}$ region to in fact be somewhat covered with $\mathrm{Na}^{+}$species in both instances, see Figure $\mathrm{S} 11$.

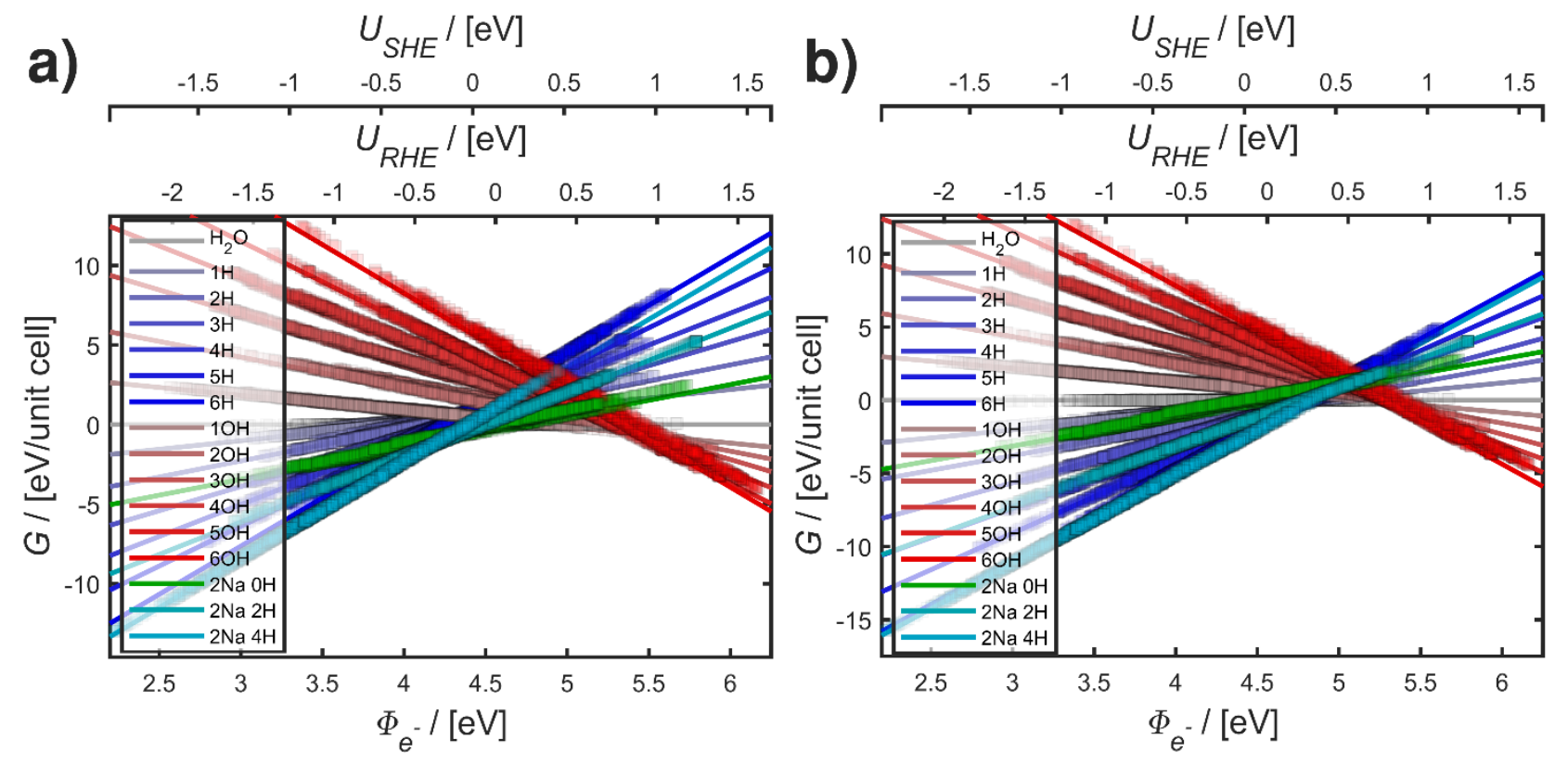

Figure S11. AIMD simulations of the $\mathrm{Pt}(111)$ interface energies at different coverages ( $N$-values) under acidic conditions ant $\mathrm{pH} 1.2$ and $\left[\mathrm{Na}^{+}\right]=0.1 \mathrm{M}$; a) Raw AIMD simulation; b) Fitted values, all Na-states have been shifted according to that expected for alkaline conditions. 
References

(1) Bagger, A. Theoretical Electrocatalysis-CO2 Redcution and the Interface, University of Copenhagen, 2019.

(2) Escudero-Escribano, M.; Pedersen, A. F.; Ulrikkeholm, E. T.; Jensen, K. D.; Hansen, M. H.; Rossmeisl, J.; Stephens, I. E. L.; Chorkendorff, I. Active-Phase Formation and Stability of Gd/Pt(111) Electrocatalysts for Oxygen Reduction: An In Situ Grazing Incidence X-Ray Diffraction Study. Chem. - A Eur. J. 2018, No. 111, 1-12.

(3) Trasatti, S. The Absolute Electrode Potential: An Explanatory Note (Recommendations 1986). Pure Appl. Chem. 1986, 58 (7), 955-966.

(4) Bard, A. J.; Faulkner, L. R. Electrochemical Methods: Fundamentals and Application, 2nd ed.; Wiley: New York, 2001.

(5) Clavilier, J.; Faure, R.; Guinet, G.; Durand, R. Preparation of Monocrystalline Pt Microelectrodes and Electrochemical Study of the Plane Surfaces Cut in the Direction of the $\{111\}$ and $\{110\}$ Planes. J. Electroanal. Chem. 1979, 107 (1), 205-209.

(6) Jensen, K. D.; Tymoczko, J.; Rossmeisl, J.; Bandarenka, A. S.; Chorkendorff, I.; Escudero-Escribano, M.; Stephens, I. E. L. Elucidation of the Oxygen Reduction Volcano in Alkaline Media Using a Copper-Platinum(111) Alloy. Angew. Chemie Int. Ed. 2018, 57 (11), 2800-2805.

(7) Pedersen, C. M.; Escudero-Escribano, M.; Velázquez-Palenzuela, A.; Christensen, L. H.; Chorkendorff, I.; Stephens, I. E. L. Benchmarking Pt-Based Electrocatalysts for Low Temperature Fuel Cell Reactions with the Rotating Disk Electrode: Oxygen Reduction and Hydrogen Oxidation in the Presence of $\mathrm{CO}$ (Review Article). Electrochim. Acta 2015, $179(0), 647-657$.

(8) Strmcnik, D. S.; Wang, C.; Stamenkovic, V. R.; Markovic, N. M.; Koper, M. T. M.; Vliet, D. Van Der; Strmcnik, D. S.; Wang, C.; Stamenkovic, V. R.; Markovic, N. M.; 
et al. On the Importance of Correcting for the Uncompensated Ohmic Resistance in Model Experiments of the Oxygen Reduction Reaction. J. Electroanal. Chem. 2010, $647(1), 29-34$.

(9) Zamburlini, E.; Jensen, K. D.; Stephens, I. E. L.; Chorkendorff, I.; Escudero-Escribano, M. Benchmarking Pt and Pt-Lanthanide Sputtered Thin Films for Oxygen Electroreduction: Fabrication and Rotating Disk Electrode Measurements. Electrochim. Acta 2017, 247, 708-721.

(10) Li, M. F.; Liao, L. W.; Yuan, D. F.; Mei, D.; Chen, Y.-X. PH Effect on Oxygen Reduction Reaction at Pt(111) Electrode. Electrochim. Acta 2013, 110, 780-789.

(11) Verdaguer-Casadevall, A.; Hernandez-Fernandez, P.; Stephens, I. E. L.; Chorkendorff, I.; Dahl, S. The Effect of Ammonia upon the Electrocatalysis of Hydrogen Oxidation and Oxygen Reduction on Polycrystalline Platinum. J. Power Sources 2012, 220, 205210.

(12) Schouten, K. J. P.; van der Niet, M. J. T. C.; Koper, M. T. M. Impedance Spectroscopy of $\mathrm{H}$ and $\mathrm{OH}$ Adsorption on Stepped Single-Crystal Platinum Electrodes in Alkaline and Acidic Media. Phys. Chem. Chem. Phys. 2010, 12 (46), 15217.

(13) Rizo, R.; Sitta, E.; Herrero, E.; Climent, V.; Feliu, J. M. Towards the Understanding of the Interfacial PH Scale at Pt(1 11 1) Electrodes. Electrochim. Acta 2015, 162, 138-145.

(14) Rizo, R.; Herrero, E.; Feliu, J. M. Oxygen Reduction Reaction on Stepped Platinum Surfaces in Alkaline Media. Phys. Chem. Chem. Phys. 2013, 15 (37), 15416.

(15) Arán-Ais, R. M.; Figueiredo, M. C.; Vidal-Iglesias, F. J.; Climent, V.; Herrero, E.; Feliu, J. M. On the Behavior of the Pt(100) and Vicinal Surfaces in Alkaline Media. Electrochim. Acta 2011, 58 (1), 184-192.

(16) Sebastián, P.; Martínez-Hincapié, R.; Climent, V.; Feliu, J. M. Study of the Pt (111)| Electrolyte Interface in the Region Close to Neutral PH Solutions by the Laser Induced Temperature Jump Technique. Electrochim. Acta 2017, 228, 667-676. 
(17) Ledezma-Yanez, I.; Wallace, W. D. Z.; Sebastián-Pascual, P.; Climent, V.; Feliu, J. M.; Koper, M. T. M. M. Interfacial Water Reorganization as a PH-Dependent Descriptor of the Hydrogen Evolution Rate on Platinum Electrodes. Nat. Energy 2017, 2 (4), 1-7.

(18) Briega-Martos, V.; Herrero, E.; Feliu, J. M. Effect of PH and Water Structure on the Oxygen Reduction Reaction on Platinum Electrodes. Electrochim. Acta 2017, 241, 497-509. 